Relations industrielles

Industrial Relations

\title{
Le Congrès 1962 de la C.S.N. : l'Action politique
}

\section{Gilles Beausoleil}

Volume 18, numéro 1, janvier 1963

URI : https://id.erudit.org/iderudit/1021456ar

DOI : https://doi.org/10.7202/1021456ar

Aller au sommaire du numéro

Éditeur(s)

Département des relations industrielles de l'Université Laval

ISSN

0034-379X (imprimé)

1703-8138 (numérique)

Découvrir la revue

Citer ce document

Beausoleil, G. (1963). Le Congrès 1962 de la C.S.N. : l'Action politique. Relations industrielles / Industrial Relations, 18(1), 80-85.

https://doi.org/10.7202/1021456ar

Tous droits réservés (C Département des relations industrielles de l’Université Laval, 1963
Ce document est protégé par la loi sur le droit d'auteur. L’utilisation des services d'Érudit (y compris la reproduction) est assujettie à sa politique d'utilisation que vous pouvez consulter en ligne.

https://apropos.erudit.org/fr/usagers/politique-dutilisation/ 


\section{COMMENTAIRES}

\section{LE CONGRES 1962 DE LA C.S.N.: L'ACTION POLITIQUE}

\section{Girles Benusolem}

Le dernier Congrès de la Confédération des Syndicats Nationaux a été témoin d'un réalignement majeur de cette centrale dans le domaine de l'action politique. Les décisions du Congrès, comprenant des modifications à la Constitution, revêtent une importance capitale. L'endossement des délégués du Congrès de la déclaration de la C.S.N. du 8 juin 1962 à la veille des élections, déclaration qui n'avait été décidée que par le Bureau Confédéral et non par le Congrès, ne fut empreint d'aucune hésitation puisque le Congrès alla plus loin en changeant la Constitution pour permettre à ses dirigeants de prendre des positions similaires à l'avenir quand ce serait désirable pour le bien-être de la classe ouvrière. De plus, la C.S.N. s'engagea à créer des comités d'action politique à tous les niveaux et permit à ses dirigeants d'endosser un parti politique lors d'élections. C'est pour ce mouvement syndical la transition définitive de l'éducation à l'action politique, cette action étant conduite avec l'aide des ressources institutionnelles et financières du mouvement et pouvant constituer une politique officielle de la Centrale.

La crise créditiste constitua apparemment un facteur de première importance des décisions capitales prises par le Congrès. Une partie considérable du Rapport du Président Général fut consacrée à la question du Crédit Social. L'argumentation de M. Marchand visait deux points: l'antisyndicalisme du Crédit Social et les carences profondes de sa théorie économique. Sur ce dernier point, la démonstration très vivante clarifie le rapport entre pouvoir d'achat et revenu national, et souligne les dangers d'une politique monétaire créditiste qui est propre à entraîner l'inflation. M. Marchand indique très nettement que la «nationalisation du crédit » est un mythe puisque le crédit est déjà contrôlé par la Banque du Canada. L’analyse des mécanismes monétaires contenue dans le rapport du Président semble avoir été suffisamment convaincante pour laisser sans moyen les tenants du Crédit Social qui étaient délégués au Congrès.

Mais la place occupée au Congrès par la question créditiste n'écarte pas la possibilité que cette question n'ait eu qu'une minime influence indirecte sur la réorientation politique de la C.S.N. Certes les résultats 
des dernières élections fédérales ont fait réaliser aux dirigeants de la C.S.N. qu'en l'absence d'une action syndicale efficace, les membres des syndicats chercheraient par eux-mêmes, des voies politiques dynamiques en dehors du syndicalisme pour apporter des solutions aux réels problèmes économiques du Québec. Ces problèmes sont sérieux et affectent presque toutes les parties de la province où se trouvent les groupes principaux de syndiqués de la C.S.N. Sauf la région métropolitaine, toutes les régions de la province sont aux prises avec une stagnation économique qui arrête la hausse des revenus ouvriers moyens. Le chômage, l'automation et la migration des travailleurs de la campagne vers les centres industriels créent chez les travailleurs une insécurité inconnue depuis la guerre. Au cours des années récentes, la hausse faible mais persistante des prix, surtout des prix des biens de nécessité, et une augmentation de la taxation directe et indirecte ont contribué à réduire le revenu réel.

Dans le rapport du Président, ces préoccupations économiques se reflètent fortement. Après avoir indiqué qu'une politique exclusive de la négociation collective n'est possible pour le syndicalisme que quand le système économique fournit un taux de croissance adéquat, $M$. Marchand indique que cette situation n'est plus celle que connaît le Canada depuis plusieurs années et qu'en conséquence le syndicalisme doit se reposer sur l'action politique. La brève description des données des problèmes économiques canadiens contenue dans ce rapport touche les points essentiels: l'économie canadienne a connu un développement considérable des secteurs primaire et tertiaire alors que l'augmentation du standard de vie dans une économie de plein-emploi est fonction de la prospérité du secteur secondaire et/ou de termes d'échange favorables sur le marché extérieur. Si l'économie canadienne ne veut pas demeurer stagnante tout en faisant face au chômage chronique, des décisions économiques de toute première importance doivent être prises au plus tôt et ces décisions reposent entre les mains des Canadiens, et non pas des Américains ou des Britanniques. Pour que ces décisions puissent être effectuées de façon sérieuse, l'économie canadienne doit recourir au plus tôt à la planification.

Sur ce point, la C.S.N. se retrouve sur le même banc que le Congrès du Travail du Canada. Le syndicalisme canadien prône de telles mesures depuis plusieurs années. Est-ce à dire que l'action politique de la C.S.N. se fera dans le même sens que celle du C.T.C. qui est officiellement affilié au Nouveau Parti Démocratique? Cette option n'a pas été faite au dernier Congrès et on peut arguer qu'il se pose des options plus pressantes que celle-ci du moins à l'échelle du milieu canadien-français. La C.S.N. se doit de développer rapidement une pensée cohérente propre à influencer une politique économique efficace du point de vue du bien-être des classes populaires. Dans la plupart des pays du monde où l'Etat développe une politique économique, soit seul soit de concert avec le grand capitalisme, une telle tâche n'est pas nécessaire, le syndicalisme se limitant à jouer le rôle de l'opposition; si cette opposition est 
efficace, la répartition du revenu national peut ne pas être défavorable aux travailleurs. Mais dans la province de Québec, l'Etat n'en est qu'aux balbutiements dans l'ordre de la politique économique et le milieu canadien-français est dépourvu de grand capitalisme. Par contre, la politique économique fédérale a tendu sans vergogne à avantager l'Ontario et l'Ouest du pays. Les partis d'opposition, y compris le N.P.D., ne tendent pas à développer des politiques économiques sérieuses, réalisables et efficaces tant à l'échelle provinciale que fédérale. Les seules forces motrices dans l'élaboration d'une telle politique sont constituées par la grande entreprise anglo-saxonne, les groupements agricoles de l'Ouest du pays et le syndicalisme. Si le syndicalisme ne participe à l'élaboration de la politique économique, ce seront les autres groupes qui s'en chargeront. L'erreur britannique devrait ici servir de leçon; pour ne s'en être tenu qu'à un rôle d'opposition politique, le syndicalisme a laissé définir la politique économique seulement par l'Etat et la grande entreprise. Dans le cas des Canadiens-français, ce serait une erreur tragique car nous n'avons pas de capitalisme; le résultat serait que d'autres définiront pour nous notre politique économique si le syndicalisme se laisse enfermer dans une position de stérile opposition.

L'objectif immédiat de l'action politique du syndicalisme à la C.S.N. consiste dans l'établissement d'organismes de planification dans le cadre de l'économie canadienne. Ce fut là le leitmotiv du Congrès. Deux aspects de ce problème se posent, celui de l'organisation institutionnelle de la planification et celui des politiques économiques en préparation du plan ou à l'intérieur de celui-ci. Sur la première question, peu de précisions furent apportées sauf sur le caractère impératif de la planification où l'Etat devrait posséder un assez large pouvoir d'intervention. Il était très correct de la part du Congrès de la C.S.N. de ne pas accorder beaucoup d'importance à l'organisation institutionnelle de la planification car ce qui compte, ce ne sont pas le nombre et le rôle des commissions à l'intérieur du Plan comme le laisse croire le récent rapport du Conseil d'Orientation Economique mais les décisions prises à l'intérieur du Plan.

Le syndicalisme a d'excellentes raisons de ne pas être indifférent au contenu des décisions de planification car elles sont toutes de nature à affecter le niveau d'emploi et le standard de vie de la classe ouvrière. Il y a diverses voies possibles de développement économique et certaines de ces voies comportent un coût social élevé pour les classes populaires. Il n'est aucunement certain à priori que l'Etat optera pour les meilleures solutions du point de vue du bien-être des travailleurs; le syndicalisme doit avoir une présence constante et éclairée pour assurer que les sacrifices relatifs des travailleurs ne seront pas plus considérables que ceux des autres groupes de la société et que leur rationalité soit telle qu'en longue période ces sacrifices seront compensés par des gains se soldant par une hausse du standard de vie. Car il serait certes très illusoire et décevant de penser qu'un plan économique prévoyant 
une intervention réelle telle que prônée par le syndicalisme ne touchera que les profits des «méchants capitalistes » sans limiter l'action du syndicalisme. Au contraire, dans la plupart des plans, souvent une plus grande attention est apportée au contrôle des salaires qu'au surplus économique des entreprises. Les interventions du Président de la C.S.N. à nombre de reprises indiquent que les dirigeants syndicaux sont conscients de ces dimensions de la planification. Aussi devra-t-on non seulement surveiller le plan mais participer activement à son élaboration pour que les objectifs traditionnels du syndicalisme soient respectés.

Sur le plan d'une pensée propre à servir de base à une politique économique sérieuse, les travaux du Congrès de la C.S.N. furent plutôt décevants. Une contribution valable aurait pu se trouver dans les rapports spéciaux et les résolutions présentés au Congrès, à part le cas du Rapport du Président de la C.S.N.; les commentaires faits plus haut indiquent assez bien l'excellence que nous attribuons à ce rapport. Mais on ne peut dire trop de bien du plan d'un régime d'allocations de retraite et surtout de l'étude portant sur le syndicat et l'automation. Dans le premier cas, si on a bien exposé les objectifs d'un plan général d'allocations de retraite, l'analyse des divers systèmes était plutôt réduite et les problèmes économiques de la transformation d'assurances sociales privées en assurances publiques n'ont pas été touchées. ${ }^{1}$ Mais le rapport sur l'automation est beaucoup plus discutable que le précédent. En premier lieu, cette étude se donne des prétentions de recherches alors que la contribution de la recherche y est mince et exposée d'une façon qu'il n'est pas possible de vérifier les méthodes employées pour conduire la recherche concernée. L'auteur ou les auteurs semblent ignorer l'abondante littérature nord-américaine et certains travaux canadiens portant sur l'automation. L'analyse est superficielle, générale et fait preuve d'une absence déplorable de connaissance économique. En dernier lieu, les solutions proposées indiquent une propension poussée vers l'utilisation de moyens légaux pour solutionner des problèmes économiques, ce qui ne constitue pas toujours la méthode la plus heureuse et la plus efficace.

Parmi les résolutions acceptées par le Congrès, la Résolution No 118 portant sur l'orientation politique constitua une résolution-clef du Congrès. Après avoir établi en priorité la mise sur pieds d'un plan économique, cette résolution propose un certain nombre de politiques économiques pour ramener l'économie canadienne au plein-emploi et lui faire retrouver un taux de croissance raisonnable. Au chapitre du plein-emploi, l'on se contente de réclamer de l'Etat l'établissement d'un niveau d'emploi élevé. C'est très édifiant de réclamer cela mais comme l'Etat ne semble pas avoir grand succès sur ce point, il faudrait reconnaître d'abord qu'il s'agit d'un problème très complexe et non pas d'un

(1) Une présentation moins brève de ce problème est contenue dans un article récent de l'auteur: Gilles Beausoleil, Syndicalisme et Sécurité Sociale, Bien-être Social Canadien, vol. 14, no 5, novembre-décembre 1962. 
complot ourdi par les méchants et en second lieu, il faudrait être beaucoup plus explicite car il y a bien des manières d'arriver au plein-emploi. Certes, plusieurs autres paragraphes de cette résolution sont conçus comme proposant des instruments propres à assurer le plein-emploi. L'élargissement du secteur public constitue une de ces mesures. Certes, dans le cas du Québec, l'accroissement de l'investissement public s'impose après quinze années de famine sur ce plan; l'éducation, entre autres, se doit de recevoir des ressources réelles plus considérables. Mais l'accroissement de l'investissement social doit être rationnel, efficace et sélectif et n'implique pas du tout l'élargissement du secteur public. Il n'est certes pas certain qu'un chèque en blanc à ce secteur soit désirable car, à partir d'indices évidents, son inefficacité économique chez-nous si ce n'est dans l'ensemble de l'Amérique du Nord est suffisamment apparente pour se passer de commentaires. La seule dimension de ce secteur, le caractère complexe de son organisation, les politiques rétrogrades de personnel et de salaires, le conformisme imposé aux fonctionnaires en font un secteur difficilement dynamique. Comme la revalorisation de la fonction publique n'est pas chose faite, il serait préférable de laisser l'Etat digérer l'assurance-hospitalisation, la sécurité sociale et la nationalisation de l'électricité avant de vouloir lui attribuer un champ plus large dans l'intervention sur des marchés ou dans la vente de biens et services.

L'extension de la sécurité sociale, une aide accrue à l'habitation et l'éducation gratuite à tous les niveaux constituent des mesures qui soulèvent nombre de problèmes. En premier lieu, la plupart de ces mesures impliquent un accroissement considérable de dépenses de consommation, ce qui ne peut stimuler l'économie qu'en compagnie d'investissements élevés si l'on veut assurer le plein-emploi. Il n'est pas précisé d'où va venir l'épargne pour assurer la hausse des investissements si l'on accroît en même temps la consommation et on ne semble pas soupçonner que de telles augmentations de consommation et d'investissements sont propres à aggraver le problème canadien de la balance des paiements. En second lieu, on peut se demander si l'extension intelligente et sélective de l'assistance sociale n'est pas plus pressante que l'élargissement d'un système massif de sécurité sociale et si l'aide à l'habitation n'a pas plus servi à permettre à la classe moyenne de se payer de meilleures habitations qu'aux pauvres à obtenir des maisons tenant compte de leurs besoins. Aussi ces points devraient-ils être examinés attentivement avant de recommander un accroissement considérable de la sécurité sociale et de l'aide à l'habitation. Quant à l'éducation gratuite, on peut se demander si aux niveaux secondaire et universitaire, un système généralisé de bourses pour les jeunes qui en ont véritablement besoin ne serait pas préférable à ce qu'on entend par l'éducation gratuite. ${ }^{2}$

(2) Voir l'article indiqué plus haut. 
Sur le plan du commerce extérieur, on recommande des mesures pour accroître le commerce extérieur et une assistance canadienne aux pays sous-développés de $2 \%$ du revenu national. Ces objectifs sont désirables en autant qu'il s'agit d'accroissement des exportations et non des importations et que l'aide consiste dans des biens fabriqués au Canada qui ne soient pas des moyens de subventionner indirectement des industries marginales. Ces mesures mettent en cause la politique commerciale et tarifaire qui est désirable pour le Canada mais ne contiennent aucune proposition précise quant à ce que devrait être cette politique.

Le postulat de base de notre évaluation du contenu de la pensée économique syndicale consiste dans la proposition que le syndicalisme doit jouer un rôle positif dans l'élaboration de la politique économique, particulièrement dans le contexte du Canada français, puisque les effets d'une planification toucheront le niveau de vie de la classe ouvrière. Sur ce point, la contribution du Congrès de la C.S.N. fut plutôt faible. Ce n'est pas là un blâme pour cette centrale syndicale. Au moins y a-t-on posé des problèmes importants en termes analytiques valables tandis que le rapport du Conseil d'Orientation Economique basé sur des moyens plus considérables et un accès plus grand à l'expertise économique n'a inclus que des généralités et même des planitudes quand ce ne sont pas des propositions qui ne sont pas prouvées telle que la suivante: "lintégration poussée de l'économie canadienne à l'économie américaine est responsable dans une large mesure de la paresse de l'économie canadienne $\gg .^{3}$ Sur ce plan, les travaux de contenu économique du Congrès de la C.S.N. souffrent bien la comparaison.

\section{LE RAPPORT GILL}

\section{Jacques St-Laurent}

1 - L'intérêt du Rapport Gill ${ }^{1}$ est triple: il permet d'apprécier rapidement l'évolution du régime d'assurance-chômage au cours de ses vingt et une années d'existence; il présente certaines statistiques qui soulignent de façon dramatique l'accroissement du chômage depuis 1954-55; il offre enfin des suggestions intéressantes concernant le règlement des problèmes financiers des chômeurs.

2 - Fondamentalement, les suggestions des commissaires consistent en un ré-arrangement du fardeau financier occasionné par la nécessité d'assurer un revenu à certains de ceux qui n'ont pas de travail. De

(3) Le Conseil d'Orientation Economique du Québec, Documents de base en vue de la planification $\gg$, reçus et adoptés en essemblée régulière du Conseil d'Orientation Economique du Québec, le 21 septembre 1962.

(1) Rıpport du Comité d'Enquête relatif da la Loi sur lassurance-chômage, Ottawa, novembre 1962. 